Spin Physics (SPIN2014)

International Journal of Modern Physics: Conference Series

Vol. 40 (2016) 1660001 (10 pages)

(C) The Author(s)

DOI: $10.1142 /$ S2010194516600016

\title{
The Spin Structure of the Nucleon
}

\author{
Xiangdong Ji \\ INPAC, Department of Physics and Astronomy, Shanghai Jiao Tong University, \\ Shanghai, 200240, P. R. China \\ Center for High-Energy Physics, Peking University, \\ Beijing, 100080, P. R. China \\ Maryland Center for Fundamental Physics, University of Maryland, \\ College Park, MD 20742, USA \\ xji@physics.umd.edu \\ Yong Zhao \\ Maryland Center for Fundamental Physics, University of Maryland, \\ College Park, MD 20742, USA \\ yongzhao@umd.edu
}

Published 29 February 2016

\begin{abstract}
We justify the physical meaning of the spin and orbital angular momentum of free partons in the infinite momentum frame, and discuss the relationship between the JaffeManohar and Ji's sum rules for proton spin. The parton orbital angular momentum in the Jaffe-Manohar sum rule can be measured through twist-three GPD's in hard scattering processes such as deeply virtual Compton scattering. Furthermore, we propose that the paton orbital angular momentum as well as the gluon helicity can be calculated in lattice QCD through a large momentum effective theory approach, and provide all the one-loop matching conditions for the proton spin content in perturbative QCD.
\end{abstract}

Keywords: Proton spin sum rule; Twist-three GPD; Lattice QCD.

PACS numbers: 14.20.Dh, 13.88.+e, 12.38.Gc, 12.38.Bx

\section{Introduction}

It is an important goal in hadron physics to understand the spin structure of the nucleon. In 1987, the European Muon Collaboration (EMC) at CERN discovered that the quark spin only accounts for a very small portion of the longitudinal proton spin, ${ }^{1,2}$ which is far less than expected from people's understanding at that time. Since then, the EMC result has inspired generations of hadron physics programs

This is an Open Access article published by World Scientific Publishing Company. It is distributed under the terms of the Creative Commons Attribution 3.0 (CC-BY) License. Further distribution of this work is permitted, provided the original work is properly cited. 
to study the spin content of the proton. Among them are SLAC, SMC and COMPASS at CERN, HERMES at DESY, PHENIX, STAR and BRAHMS at RHIC, and JLab (see a recent review in Ref. 3). Our current understanding of the proton spin structure is that the quark spin contributes about one third, ${ }^{4}$ while there is strong evidence that the gluon polarization also shares a considerable positive amount; ${ }^{5}$ the rest is believed to be distributed between the quark and gluon orbital angular momentum (OAM). With the JLab $12 \mathrm{GeV}$ upgrade ${ }^{6}$ as well as the electron-ion collider (EIC), ${ }^{7}$ people will be able to obtain more abundant and accurate information about the structure of proton spin.

The key issues associated with the proton spin structure are:

- What is a physical sum rule for the proton spin?

- How to probe the OAM of the quark and gluon partons?

- How to calculate the gluon helicity and parton OAM in lattice QCD?

\section{The Jaffe-Manohar and Ji's Sum Rules for Proton Spin}

In the past 25 years, two well-known spin sum rules have been proposed to analyze the proton spin structure. The first, proposed by Jaffe and Manohar, ${ }^{8}$ was motivated from a free-field expression of QCD angular momentum boosted to the infinite momentum frame (IMF) of the proton. The second, usually called Ji's sum rule, is the frame-independent and manifestly gauge-invariant decomposition by one of the authors. ${ }^{9}$

The Jaffe-Manohar sum rule is defined in the light-cone gauge $A^{+}=0$, and states that the proton spin can be decomposed into four parts,

$$
\frac{1}{2}=\frac{1}{2} \Delta \Sigma(\mu)+l_{q}^{z}(\mu)+\Delta G(\mu)+l_{g}^{z}(\mu),
$$

where the individual terms are the spin and OAM of the quark and gluon partons, respectively, and $\mu$ is a renormalization scale. All the four terms are defined to be the proton matrix elements of free-field angular momentum operators in the IMF: 8

$$
\begin{aligned}
\vec{J}= & \int d^{3} x \psi^{\dagger} \frac{\vec{\Sigma}}{2} \psi+\int d^{3} x \psi^{\dagger} \vec{x} \times(-i \vec{\nabla}) \psi \\
& +\int d^{3} x \vec{E}_{a} \times \vec{A}^{a}+\int d^{3} x E_{a}^{i} \vec{x} \times \vec{\nabla} A^{i, a},
\end{aligned}
$$

where $E^{i}=F^{i+}, a$ and $i$ are the color and spatial indices. Here the system is fixed on the light-cone plane, i.e., $x^{+}=\left(x^{0}+x^{3}\right) / \sqrt{2}=0$, which is equivalent to the IMF.

In the light-cone gauge, each term in Eq. (2) can be expressed as sum of the spin and OAM over all Fock states, so the Jaffe-Manohar sum rule has a clear partonic interpretation. However, the free-field form of the angular momentum in gauge theories faces two conceptual problems: all terms except the first one are gauge dependent, and it is unclear why the light-cone gauge operator is measurable in experiments. 
Ji's sum rule takes a different form from Eq. (2), as the total QCD angular momentum is decomposed into three gauge-invariant parts: ${ }^{9}$

$$
\begin{aligned}
\vec{J}= & \int d^{3} x \psi^{\dagger} \frac{\vec{\Sigma}}{2} \psi+\int d^{3} x \psi^{\dagger} \vec{x} \times(-i \vec{\nabla}-g \vec{A}) \psi \\
& +\int d^{3} x \vec{x} \times(\vec{E} \times \vec{B}),
\end{aligned}
$$

where the total gluon angular momentum cannot be gauge-invariantly decomposed into its spin and OAM. In this way, Ji's sum rule reads:

$$
\frac{1}{2}=\frac{1}{2} \Delta \Sigma(\mu)+L_{q}^{z}(\mu)+J_{g}^{z}(\mu) .
$$

Notwithstanding that Ji's sum rule has received considerable attention for its relation to generalized parton distributions (GPD's) and experimental probes, ${ }^{9-11}$ it is not natural in the language of parton physics (see, however, a recent discussion on its connection to the transverse polarization $\left.{ }^{12}\right)$.

Since the gluon polarization is not defined from the Ji sum rule, it has generated a lot of theoretical attempts to define gauge-invariant gluon spin and OAM angular momentum operators. ${ }^{13-16}$ As has been discussed extensively in a recent review, ${ }^{17}$ these new sum rules can be classified into two classes - the Jaffe-Manohar and Ji types. There are two key issues that distinguish these two types of sum rules: the Jaffe-Manohar sum rule is frame dependent as it is defined in the IMF, but the Ji's sum rule does not depend on any frame. Besides, as one can compare Eq. (2) and Eq. (3), the Jaffe-Manohar sum rule uses the canonical OAM operators, while Ji's sum rule adopts the "mechanical" OAM operator that results from the BelinfanteRosenfeld procedure.

In the past two decades, there has been a long list of literatures attempting to justify the Jaffe-Manohar sum rule as physical (see e.g., Refs. 18, 19, 20, 21, 22, 23, 24). There are strong motivations behind this: First, $\Delta G$ as defined in the lightcone gauge is measurable in high-energy experiments, although this appears to be a theoretical puzzle by itself - while $\Delta G$ is easy to define from the Feynman parton picture, there is no natural gauge-invariant notion for the spin of gauge particles. ${ }^{25}$ Second, the simple partonic picture of the Jaffe-Manohar sum rule in the IMF makes it more natural to use free-field operators.

It was first proposed that although the free-field angular momentum operators are gauge dependent, their physical matrix elements are gauge invariant. ${ }^{19}$ A similar claim was also made recently. ${ }^{20}$ However, this is invalidated by a one-loop calculation, ${ }^{23}$ where the matrix element of the gluon spin operator was shown to be different in the Coulomb and light-cone gauges, as has been proved in Ref. 26 (for more general discussions see Ref. 21). Actually, as argued in Refs. ${ }^{22,23}$ for the bound-state proton, there is no physically meaningful notion of gluon spin or OAM due to the existence of longitudinal gluons. Only when the proton is boosted to IMF, the longitudinal component of gluons is suppressed by the infinite boost and the gluons can be regarded as free radiation. This is the well-known Weizsäcker-Williams (WW) 
approximation. ${ }^{27}$ The gluon spin then acquires a clear physical meaning and can be represented by $\vec{E} \times \vec{A}$, but is subject to a class of "physical" gauge conditions that leave the transverse polarizations of the gluon field intact. ${ }^{24}$ Similar arguments also apply to the quark and gluon OAM. Therefore, we can regard the free-field form in the Jaffe-Manohar sum rule as physical if we work in IMF with a "physical" gauge condition. This is equivalent to using the light-cone coordinates and gauge, ${ }^{8}$ and the reason is simple: All the "physical" gauges will flow into the light-cone gauge in the IMF limit. ${ }^{24}$

\section{Probing the Parton Orbital Angular Momentum}

When Ji's sum rule was first proposed, it immediately receives a lot of attention because each term can be measured through twist-two GPD's from deeply virtual Compton scattering (DVCS) experiments..$^{9,}{ }^{10}$ In Ji's sum rule, the total quark and gluon angular momenta satisfy

$$
\begin{aligned}
J_{q, g} & =\frac{1}{2}\left[A_{q, g}(0)+B_{q, g}(0)\right], \\
J_{q}+J_{g} & =\frac{1}{2},
\end{aligned}
$$

where $A_{q, g}(0)$ and $B_{q, g}(0)$ are form factors of the symmetrized quark and gluon energy-momentum tensors.

In a DVCS process as shown in Fig. 1, the Compton amplitude depends on four twist-two GPD's, $H, \tilde{H}, E$ and $\tilde{E}$. In the light-cone gauge, they are defined to be the off-forward matrix elements of the light-cone correlations:

$$
\begin{aligned}
\int \frac{d \lambda}{2 \pi} e^{i \lambda x}\left\langle P^{\prime}\left|\bar{\psi}\left(-\frac{\lambda n}{2}\right) \gamma^{\mu} \psi\left(\frac{\lambda n}{2}\right)\right| P\right\rangle= & H\left(x, \Delta^{2}, \xi\right) \bar{U}\left(P^{\prime}\right) \gamma^{\mu} U(P) \\
& +E\left(x, \Delta^{2}, \xi\right) \bar{U}\left(P^{\prime}\right) i \frac{\sigma^{\mu \nu} \Delta_{\nu}}{2 M} U(P)+\cdots, \\
\int \frac{d \lambda}{2 \pi} e^{i \lambda x}\left\langle P^{\prime}\left|\bar{\psi}\left(-\frac{\lambda n}{2}\right) \gamma^{\mu} \gamma_{5} \psi\left(\frac{\lambda n}{2}\right)\right| P\right\rangle= & \tilde{H}\left(x, \Delta^{2}, \xi\right) \bar{U}\left(P^{\prime}\right) \gamma^{\mu} U(P) \\
& +\tilde{E}\left(x, \Delta^{2}, \xi\right) \bar{U}\left(P^{\prime}\right) i \frac{\sigma^{\mu \nu} \Delta_{\nu}}{2 M} U(P)+\cdots,
\end{aligned}
$$

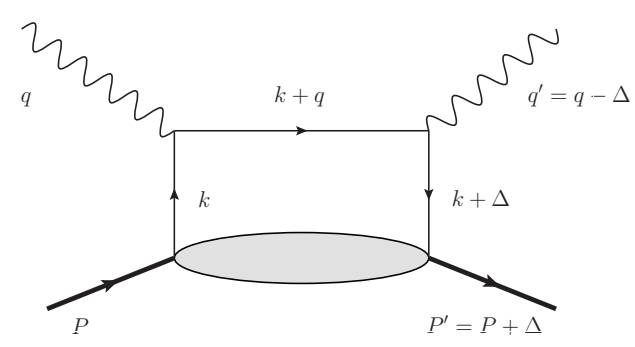

Fig. 1. Dominant scattering process in DVCS. 
where $|P\rangle$ is a plane-wave proton state with momentum $P^{\mu}=\left(P^{0}, 0,0, P\right), n^{\mu}$ is a vector along the light-cone direction, and the skewness parameter $\xi=-n \cdot \Delta / n$. $\left(P+P^{\prime}\right)$. Here the “..." represents higher-twist contributions. The proton spin is related to these GPD's through the sum rule ${ }^{9,10}$

$$
\int_{-1}^{1} d x x\left[H\left(x, \xi, \Delta^{2}\right)+E\left(x, \xi, \Delta^{2}\right)\right]=A_{q}\left(\Delta^{2}\right)+B_{q}\left(\Delta^{2}\right) .
$$

To obtain $J_{q}$, one can extrapolate the sum rule to $\Delta^{2}=0$, and then $J_{g}=1 / 2-J_{q}$. Since the quark spin $\Delta \Sigma$ has been precisely measured in inclusive and semi-inclusive scattering experiments, one can subtract it from $J_{q}$ to determine $L_{q}^{z}$ in Ji's sum rule. In the IMF, $J_{g}$ can be further decomposed into three parts,

$$
J_{g}^{z}=\Delta G+l_{g}^{z}+J_{\text {pot }}^{z},
$$

where the so called "potential" angular momentum $J_{\text {pot }}^{z}$ corresponds to the operator

$$
\vec{J}_{\text {pot }}=g \int d^{3} x \psi^{\dagger} \vec{x} \times \vec{A} \psi
$$

Although $\Delta G$ is known to be measurable, to determine $l_{g}^{z}$ in the Jaffe-Manohar sum rule one still needs to measure the contribution from $J_{\text {pot }}^{z}$, which is also the case for $l_{q}^{z}$.

Since the parton OAM involves the transverse motion of the patrons, it is intrinsically a twist-three observable. However, for $L_{q}^{z}$ in Ji's sum rule, it can be related to twist-two GPD's through rotational symmetry, because the operator is frame independent. In contrast, $l_{q}^{z}$ and $l_{g}^{z}$ in the Jaffe-Manohar sum rule do not have frame independence, and to measure them as well as $J_{\text {pot }}^{z}$ one has to rely on twist-three GPD's. ${ }^{28,30}$ The relationships between $l_{q}^{z}, l_{g}^{z}$ and $J_{\text {pot }}^{z}$ are derived in Ref. 30, and we are not going to provide the details here. Meanwhile, these twist-three GPD's have been studied and can be extracted from two-photon processes such as DVCS. ${ }^{31,32}$

\section{Lattice Calculation of the Proton Spin Content}

Although we have justified its physical meaning, the Jaffe-Manohar sum rule itself still poses difficulty for a nonperturbative lattice calculation of its individual contributions, because the explicit usage of light-cone coordinates and gauge brings real-time dependence. One may avoid this difficulty by using normal space-time coordinates with a "physical" gauge that does not involve time, and calculating with a proton at infinite momentum. However, the largest momentum attainable on the lattice with spacing $a$ is constrained by the lattice cutoff $\pi / a$.

Ji's sum rule, instead, is frame independent so that one can go off the light-cone and work in a reference frame with finite momentum. Lorentz covariance guarantees that each component in this sum rule will be the same in the IMF. Therefore, a considerable amount of lattice QCD calculations have been carried out to study 
Ji's sum rule, among which are the LHPC, ${ }^{33,34}$ SESAM, ${ }^{33}$ USQCDSF-UKQCD, ${ }^{35}$ $\mathrm{ETMC}^{36,37}$ and $\chi \mathrm{QCD}^{38}$ collaborations.

The difficulty in the lattice study of the Jaffe-Manohar sum rule can, however, be circumvented in the framework of large-momentum effective field theory (LaMET) ${ }^{39}$ proposed by one of the authors. Suppose one is to calculate some light-cone or parton observable $\mathcal{O}$. Instead of computing it directly, one defines, in the LaMET framework, a quasi-observable $\tilde{\mathcal{O}}$ that depends on a large hadron momentum $P^{z}$. In general, both $\mathcal{O}$ and $\tilde{\mathcal{O}}$ suffer from ultraviolet (UV) divergences. If $P^{z} \rightarrow \infty$ is taken prior to a UV regularization, the quasi-observable $\tilde{\mathcal{O}}$ becomes the parton observable $\mathcal{O}$ by construction. However, what one can calculate in practice is the quasi-observable $\tilde{\mathcal{O}}$ at large but finite $P^{z}$ with UV regularization imposed first. This is the case in lattice computations. The difference between $\mathcal{O}$ and $\tilde{\mathcal{O}}$ is just the order of limits. This is similar to an effective field theory set-up. The difference is that here the role of perturbative degrees of freedom is played by the large momentum of the external state, hence it cannot be arranged into a Lagrangian formalism. Nevertheless, one can bridge the quasi- and parton observables through

$$
\tilde{\mathcal{O}}\left(P^{z} / \Lambda\right)=Z\left(P^{z} / \Lambda, \mu / \Lambda\right) \mathcal{O}(\mu)+\frac{c_{2}}{\left(P^{z}\right)^{2}}+\frac{c_{4}}{\left(P^{z}\right)^{4}}+\cdots
$$

where $\Lambda$ is a UV cutoff imposed on the quasi-observable, and $c_{i}$ 's are higher-twist contributions suppressed by powers of $P^{z}$. That is, the quasi-observable $\tilde{\mathcal{O}}\left(P^{z} / \Lambda\right)$ can be factorized into the parton observable $\mathcal{O}(\mu)$ and a matching coefficient $Z$, up to power suppressed corrections. Taking the $P^{z} \rightarrow \infty$ limit does not change the infrared (IR) behavior of the observable, but only affects its UV behavior. Therefore $\mathcal{O}(\mu)$ captures all the IR physics in $\tilde{\mathcal{O}}\left(P^{z} / \Lambda\right)$, and the matching coefficient $Z$ is completely perturbative.

An explicit example of Eq. (10) is presented in Refs. 40, 41 for the case of parton distribution functions (PDFs), where the factorization formula has a convolution form, and the matching coefficients were calculated at the leading logarithmic order. Using these results, the first direct lattice calculation of the isovector sea-quark parton distributions has been available recently. ${ }^{42} \mathrm{~A}$ similar factorization formula was also proposed in Ref. 43 to extract PDFs from lattice QCD calculations based on QCD factorization of lattice "cross sections".

Within the LaMET framework, we can start with suitable quasi-observables to calculate the proton spin content. According to our discussions above, these quasi-observables can be defined as the free-field QCD AMOs in a "physical" gauge condition that has the correct WW approximation in the IMF limit. ${ }^{24} \mathrm{~A}$ possible choice of the "physical" gauge condition is the expression in terms of nonlocal operators introduced by Chen et al.:13,44

$$
\begin{aligned}
\vec{J}_{\mathrm{QCD}}= & \int d^{3} x \psi^{\dagger} \frac{\vec{\Sigma}}{2} \psi+\int d^{3} x \psi^{\dagger} \vec{x} \times\left(-i \vec{\nabla}-g \vec{A}_{\|}\right) \psi \\
& +\int d^{3} x \vec{E}_{a} \times \vec{A}_{\perp}^{a}+\int d^{3} x E_{a}^{i}(\vec{x} \times \vec{\nabla}) A_{\perp}^{i, a},
\end{aligned}
$$


where $\vec{x}$ are the spatial coordinates, and $\vec{A}$ is decomposed into a pure-gauge part $\vec{A}_{\|}$and a physical part $\vec{A}_{\perp}$ which satisfy (see also Ref. 45 )

$$
\begin{array}{r}
\partial^{i} A_{\|}^{j, a}-\partial^{j} A_{\|}^{i, a}-g f^{a b c} A_{\|}^{i, b} A_{\|}^{j, c}=0, \\
\partial^{i} A_{\perp}^{i}-i g\left[A^{i}, A_{\perp}^{i}\right]=0,
\end{array}
$$

so that each term in Eq. (11) is gauge invariant. From Eq. (12), one can show that in the Coulomb gauge $\vec{\nabla} \cdot \vec{A}=0, \vec{A}_{\perp}$ equals $\vec{A}$ order by order in perturbation theory. Therefore, Eq. (11) corresponds to choosing the Coulomb gauge as the "physical" gauge.

It has been shown in Ref. 23 that $\vec{E}_{a} \times \vec{A}_{\perp}^{a}$ in Eq. (11) is equivalent to the total gluon spin operator in the IMF limit. It is easy to see that the other nonlocal terms in Eq. (11) also have the correct WW approximation as the parton OAM. Therefore, we can choose the nonlocal operators in Eq. (11) as the quasi-observables for parton angular momentum in the LaMET approach.

The advantage of the expression in Eq. (11) is that it is time independent and thus allows for a direct calculation in lattice QCD. Suppose we evaluate the matrix elements of these quasi-observables with finite momentum $P^{z}$, we should have

$$
\frac{1}{2}=\frac{1}{2} \Delta \widetilde{\Sigma}\left(\mu, P^{z}\right)+\Delta \widetilde{G}\left(\mu, P^{z}\right)+\Delta \widetilde{L}_{q}\left(\mu, P^{z}\right)+\Delta \widetilde{L}_{g}\left(\mu, P^{z}\right)
$$

where the $P^{z}$-dependence is expected since Eq. (11) is a frame-dependent expression. ${ }^{23}$ Following the effective theory argument above, we can relate these quasi-observables to the corresponding parton observables through the following factorization formula:

$$
\begin{aligned}
\Delta \widetilde{\Sigma}\left(\mu, P^{z}\right)= & \Delta \Sigma(\mu) \\
\Delta \widetilde{G}\left(\mu, P^{z}\right)= & z_{q g} \Delta \Sigma(\mu)+z_{g g} \Delta G(\mu)+O\left(\frac{M^{2}}{\left(P^{z}\right)^{2}}\right) \\
\Delta \widetilde{L}_{q}\left(\mu, P^{z}\right)= & P_{q q} \Delta L_{q}(\mu)+P_{g q} \Delta L_{g}(\mu) \\
& +p_{q q} \Delta \Sigma(\mu)+p_{g q} \Delta G(\mu)+O\left(\frac{M^{2}}{\left(P^{z}\right)^{2}}\right), \\
\Delta \widetilde{L}_{g}\left(\mu, P^{z}\right)= & P_{q g} \Delta L_{q}(\mu)+P_{g g} \Delta L_{g}(\mu) \\
& +p_{q g} \Delta \Sigma(\mu)+p_{g g} \Delta G(\mu)+O\left(\frac{M^{2}}{\left(P^{z}\right)^{2}}\right),
\end{aligned}
$$

where $M$ is the proton mass, and all the matrix elements are renormalized in the $\overline{\mathrm{MS}}$ scheme. $\Delta \widetilde{\Sigma}\left(\mu, P^{z}\right)$ is the same as $\Delta \Sigma(\mu)$ because the quark spin operator is frame independent and has the same matrix elements in the Coulomb and lightcone gauges. The $z_{i j}, P_{i j}$ and $p_{i j}$ 's are the matching coefficients to be calculated in perturbative QCD. 
All the matching coefficients in Eq. (14) at one-loop order has been calculated in Ref. 46:

$$
\begin{array}{ll}
P_{q q}=1+\frac{\alpha_{S} C_{F}}{4 \pi}\left(-2 \ln \frac{\left(P^{z}\right)^{2}}{\mu^{2}}+R_{3}\right), & P_{g q}=0, \\
P_{q g}=\frac{\alpha_{S} C_{F}}{4 \pi}\left(2 \ln \frac{\left(P^{z}\right)^{2}}{\mu^{2}}-R_{3}\right), & P_{g g}=1, \\
p_{q q}=\frac{\alpha_{S} C_{F}}{4 \pi}\left(-\frac{1}{3} \ln \frac{\left(P^{z}\right)^{2}}{\mu^{2}}+R_{4}\right), \quad p_{g q}=0, \\
p_{q g}=\frac{\alpha_{S} C_{F}}{4 \pi}\left(-\ln \frac{\left(P^{z}\right)^{2}}{\mu^{2}}-R_{1}-R_{4}\right), \\
p_{g g}=\frac{\alpha_{S} C_{A}}{4 \pi}\left(-\frac{7}{3} \ln \frac{\left(P^{z}\right)^{2}}{\mu^{2}}-R_{2}\right),
\end{array}
$$

where

$$
\begin{aligned}
C_{F}=\left(N_{c}^{2}-1\right) / 2 N_{c}, C_{A} & =N_{c}, \\
R_{1} & =\frac{8}{3} \ln 2-\frac{64}{9}, \quad R_{2}=\frac{14}{3} \ln 2-\frac{121}{9}, \\
R_{3} & =-4 \ln 2+\frac{28}{3}, \quad R_{4}=-\frac{2}{3} \ln 2+\frac{13}{9},
\end{aligned}
$$

with $N_{c}$ being the number of colors.

With the above results, we are able to convert the quasi-observables in Eq. (11) evaluated at a large finite momentum to the parton spin and OAM in IMF. This can be done by a simple inversion of Eq. (14). Although for realistic lattice computations the above matching coefficients have to be recalculated using lattice perturbation theory, ${ }^{47}$ the leading logarithmic term of the matching coefficients is independent of the regularization scheme, and therefore is the same in dimensional and lattice regularizations. Our one-loop matching coefficients can thus be used for an approximate lattice computation of parton angular momentum to leading logarithmic accuracy.

In summary, we have justified the physical significance of the Jaffe-Manohar spin sum rule as a result of the WW approximation in the IMF. The key distinction between the Jaffe-Manohar and Ji's sum rules is that the former is defined in the IMF while the latter is frame independent. The quark and gluon OAM in the Jaffe-Manohar sum rule can be measured through twist-three observables from two-photon processes such as DVCS. In addition, we have shown how to obtain the partonic contributions to proton spin using the LaMET approach. The solution is a perturbative factorization formula that allows us to extract the parton spin and OAM in the IMF from lattice QCD calculations with a finite but large proton momentum. With such developments, we can eventually compare the parton spin and OAM in theory and experiment.

We thank J. -W. Chen for useful discussions about the gauge-invariant gluon spin operator. We also thank M. Glatzmaier and K. -F. Liu for discussions on 
matching in lattice perturbation theory. This work was partially supported by the U.S. Department of Energy Office of Science, Office of Nuclear Physics under Award Number DE-FG02-93ER-40762 and a grant (No. 11DZ2260700) from the Office of Science and Technology in Shanghai Municipal Government, and grants by the National Science Foundation of China (No. 11175114, No. 11405104).

\section{References}

1. J. Ashman et al. [European Muon Collaboration], Phys. Lett. B 206, 364 (1988).

2. J. Ashman et al. [European Muon Collaboration], Nucl. Phys. B 328, 1 (1989).

3. C. A. Aidala, S. D. Bass, D. Hasch and G. K. Mallot, Rev. Mod. Phys. 85, 655 (2013) [arXiv:1209.2803 [hep-ph]].

4. D. de Florian, R. Sassot, M. Stratmann and W. Vogelsang, Phys. Rev. D 80, 034030 (2009) [arXiv:0904.3821 [hep-ph]].

5. D. de Florian, R. Sassot, M. Stratmann and W. Vogelsang, Phys. Rev. Lett. 113, no. 1, 012001 (2014) [arXiv:1404.4293 [hep-ph]].

6. J. Dudek, R. Ent, R. Essig, K. S. Kumar, C. Meyer, R. D. McKeown, Z. E. Meziani and G. A. Miller et al., Eur. Phys. J. A 48, 187 (2012) [arXiv:1208.1244 [hep-ex]].

7. A. Accardi, J. L. Albacete, M. Anselmino, N. Armesto, E. C. Aschenauer, A. Bacchetta, D. Boer and W. Brooks et al., arXiv:1212.1701 [nucl-ex].

8. R. L. Jaffe and A. V. Manohar, Nucl. Phys. B 337, 509 (1990).

9. X. -D. Ji, Phys. Rev. Lett. 78, 610 (1997) [hep-ph/9603249].

10. X. D. Ji, Phys. Rev. D 55, 7114 (1997) [hep-ph/9609381].

11. P. Hoodbhoy, X. D. Ji and W. Lu, Phys. Rev. D 59, 014013 (1998) [hep-ph/9804337].

12. X. Ji, X. Xiong and F. Yuan, Phys. Lett. B 717, 214 (2012) [arXiv:1209.3246 [hep-ph]].

13. X. S. Chen, X. F. Lu, W. M. Sun, F. Wang and T. Goldman, Phys. Rev. Lett. 100, 232002 (2008) [arXiv:0806.3166 [hep-ph]].

14. M. Wakamatsu, Phys. Rev. D 83, 014012 (2011) [arXiv:1007.5355 [hep-ph]].

15. Y. Hatta, Phys. Rev. D 84, 041701 (2011) [arXiv:1101.5989 [hep-ph]].

16. B. H. Zhou and Y. C. Huang, Phys. Rev. D 84, 047701 (2011).

17. E. Leader and C. Lorc, Phys. Rept. 541, 163 (2014) [arXiv:1309.4235 [hep-ph]].

18. R. L. Jaffe, Phys. Lett. B 365, 359 (1996) [hep-ph/9509279].

19. X. -S. Chen and F. Wang, hep-ph/9802346; F. Wang, W. -M. Sun and X. -F. Lu, hep-ph/0510285.

20. E. Leader, Phys. Rev. D 83, 096012 (2011) [Erratum-ibid. D 85, 039905 (2012)] [arXiv:1101.5956 [hep-ph]].

21. P. Hoodbhoy and X. D. Ji, Phys. Rev. D 60, 114042 (1999) [hep-ph/9908275].

22. X. Ji, Y. Xu, and Y. Zhao, JHEP 1208, 082 (2012).

23. X. Ji, J. H. Zhang and Y. Zhao, Phys. Rev. Lett. 111, 112002 (2013) [arXiv:1304.6708 [hep-ph]].

24. Y. Hatta, X. Ji and Y. Zhao, Phys. Rev. D 89, 085030 (2014) [arXiv:1310.4263 [hep$\mathrm{ph}]]$.

25. V. B. Berestetskii, L. P. Pitaevskii, and E.M. Lifshitz, "Quantum Electrodynamics," Second Edition: Volume 4 in Courses in Theoretical Physics, Butterworth-Heineman, 1982.

26. X. -D. Ji, J. Tang and P. Hoodbhoy, Phys. Rev. Lett. 76, 740 (1996) [hep-ph/9510304].

27. J. D. Jackson, Classical Electrodynamics (John Wiley \& Sons, New York, 1999), 3rd ed..

28. Y. Hatta, Phys. Lett. B 708, 186 (2012) [arXiv:1111.3547 [hep-ph]]. 
29. X. Ji, X. Xiong and F. Yuan, Phys. Rev. D 88, no. 1, 014041 (2013) [arXiv:1207.5221 [hep-ph]].

30. X. Ji, X. Xiong and F. Yuan, Phys. Rev. Lett. 109, 152005 (2012) [arXiv:1202.2843 [hep-ph]].

31. I. V. Anikin, B. Pire and O. V. Teryaev, Phys. Rev. D 62, 071501 (2000) [hep-ph/ 0003203].

32. A. V. Belitsky and D. Mueller, Nucl. Phys. B 589, 611 (2000) [hep-ph/0007031].

33. P. Hagler et al. [LHPC and SESAM Collaborations], Phys. Rev. D 68, 034505 (2003) [hep-lat/0304018].

34. J. D. Bratt et al. [LHPC Collaboration], Phys. Rev. D 82, 094502 (2010) [arXiv:1001. 3620 [hep-lat]].

35. D. Brommel et al. [QCDSF-UKQCD Collaboration], PoS LAT 2007, 158 (2007) [arXiv:0710.1534 [hep-lat]].

36. C. Alexandrou, M. Constantinou, S. Dinter, V. Drach, K. Jansen, C. Kallidonis and G. Koutsou, Phys. Rev. D 88, no. 1, 014509 (2013) [arXiv:1303.5979 [hep-lat]].

37. C. Alexandrou, J. Carbonell, M. Constantinou, P. A. Harraud, P. Guichon, K. Jansen, C. Kallidonis and T. Korzec et al., Phys. Rev. D 83, 114513 (2011) [arXiv:1104.1600 [hep-lat]].

38. M. Deka, T. Doi, Y. B. Yang, B. Chakraborty, S. J. Dong, T. Draper, M. Glatzmaier and M. Gong et al., Phys. Rev. D 91, no. 1, 014505 (2015) [arXiv:1312.4816 [hep-lat]].

39. X. Ji, Sci. China Phys. Mech. Astron. 57, no. 7, 1407 (2014) [arXiv:1404.6680 [hep$\mathrm{ph}]]$.

40. X. Ji, Phys. Rev. Lett. 110, 262002 (2013) [arXiv:1305.1539 [hep-ph]].

41. X. Xiong, X. Ji, J. H. Zhang and Y. Zhao, Phys. Rev. D 90, 014051 (2014) [arXiv:1310.7471 [hep-ph]].

42. H. -W. Lin, J. -W. Chen, S. D. Cohen and X. Ji, arXiv:1402.1462 [hep-ph].

43. Y. Q. Ma and J. W. Qiu, arXiv:1404.6860 [hep-ph].

44. X. -S. Chen, W. -M. Sun, X. -F. Lü, F. Wang, and T. Goldman, Phys. Rev. Lett. 103, 062001 (2009).

45. R. P. Treat, J. Math. Phys. 13, 1704 (1972) [Erratum-ibid. 14, 1296 (1973)].

46. X. Ji, J. H. Zhang and Y. Zhao, Phys. Lett. B 743, 180 (2015) [arXiv:1409.6329 [hep-ph]].

47. S. Capitani, Phys. Rept. 382, 113 (2003) [hep-lat/0211036]. 